the migration of neutrophils into the area of infection ${ }^{7}$. As outlined above, binding of $\mathrm{C} 3 \mathrm{~b}$ and $\mathrm{iC} 3 \mathrm{~b}$ to the appropriate phagocyte receptor is required for phagocytosis. But a capsule composed of hyaluronic acid acts as a physical barrier to prevent this interaction and thus renders the bacterium difficult to phagocytize, creating a fifth level of defense ${ }^{8}$.

As if these mechanisms of protection were not enough, Lei et $a l^{1}$. report yet another line of defense. They have discovered that group A streptococci in log-phase growth secrete a protein that has sequence homology with CD11b, the $\alpha$-chain of CR3, the receptor on phagocytes that recognizes iC $3 \mathrm{~b}$. This secreted material, which is known as Mac or Mac-1, binds to and blocks CD11b and also blocks the activity of FcrRIII (CD16) which is one of the receptors that recognizes immunoglobulin $\mathrm{G}$ (IgG). Mac also appears to bind directly to CD16. This IgG receptor is a low-affinity receptor tethered to the cell membrane by a phosphatidylinositol bond. Some of membrane CR3 is linked to FcyRIII in the cell membrane, and in many systems, blocking CR3 will not only block phagocytosis mediated by iC3b, but also IgG-mediated phagocytosis $^{9,10}$. Thus, the bacteria secrete a product (Mac) that is able to block phagocytosis by two important opsonins in serum, iC $3 \mathrm{~b}$ and $\mathrm{IgG}$, and also blocks some of the downstream results of receptor activation ${ }^{11}$.

As animals have evolved increasingly sophisticated mechanisms for fending off microbial invasion, so too have microbes evolved increasingly sophisticated mechanisms to avoid these processes. Effective host immunity represents a delicate balance between the sophisticated self-defense mechanisms of the aggressor and the concerted attack of our host-defense molecules and cells. And as we learn more about the elements of bacterial aggression, we become far more able to determine the types of vaccines and drugs that will be required to prevent successful attack.

1. Lei, B. et al. Evasion of human innate and acquired immunity by a bacterial homolog of CD11b that inhibits opsonophagocytosis. Nature Med. 7, 1298-1305 (2001).

2. Volanakis, J.E. Overview of the complement system. in The Human Complement System in Health and Disease (eds. Volanakis, J.E. \& Frank, M.M.) 9-32 (Marcel Dekker, New York, 1998).

3. Whitnack, E., Dale, J.B. \& Beachey, E.H. Common protective antigens of group A streptococcal $M$ proteins masked by fibrinogen. J. Exp. Med. 159, 1201-1212 (1984).

4. Berge, A., Kihlberg, B.M., Sjöholm, A.G. \& Björck, L. Streptococcal protein $\mathrm{H}$ forms soluble complement-activating complexes with IgG, but inhibits complement activation by IgG-coated targets. J. Biol. Chem. 272, 20774-20781 (1997).

5. Perez-Caballero, D., Alberti, S., Vivanco, F., Sanchez-Corral, P. \& Rodriguez de Cordoba, S. Assessment of the interaction of human complement regulatory proteins with group A Streptococcus. Identification of a high-affinity group A Streptococcus binding site in FHL-1. Eur. J. Immunol. 30, 1243-1253 (2000).

6. Fernie-King, B.A. et al. Streptococcal inhibitor of complement (SIC) inhibits the membrane-attack complex by preventing uptake of C567 onto cell membranes. Immunology 103, 390-398 (2001).

7. Cleary, P.P., Prahbu U., Dale J.B., Wexler D.E., \& Hadley, J. Streptococcal C5a peptidase is a highly specific endopeptidase. Infect. Immun. 60, 5219-5223 (1992).

8. Dale, J.B., Washburn, R.G., Marques, M.B. \& Wessels, M.R. Hyaluronate capsule and surface $M$ protein in resistance to opsonization of group A streptococci. Infect. Immun. 64, 1495-1501 (1996).

9. Zhou, M \& Brown, E. CR3 (Mac-1, $\alpha_{M} \beta_{2}$ $\mathrm{CD} 11 \mathrm{~b} / \mathrm{CD} 18)$ and $\mathrm{FC} \gamma \mathrm{RIII}$ cooperate in generation of a neutrophil respiratory burst: Requirement for Fc $\gamma \mathrm{RII}$ and tyrosine phosphorylation. J. Cell Biol. 125, 1407-1416 (1994).

10. Todd, R.F. III \& Petty, H.R. $\beta_{2}$ (CD11/CD18) integrins can serve as signaling partners for other leukocyte receptors. J. Lab. Clin. Med. 129, 492-498 (1997)

11. Cuzzola, M. et al. $\beta_{2}$ integrins are involved in cytokine responses to whole gram-positive bacteria. J. Immunol. 164, 5871-5876 (2000).

Department of Pediatrics

Duke Medical Center and

Children's Health Center

Durham, North Carolina, USA

Email:frank007@mc.duke.edu

\title{
Death by influenza virus protein
}

The identification of a new influenza virus protein PB1-F2, which localizes to mitochondria and causes cell death, may offer important insights into pathogenicity, as well as providing another example of how the virus can expand the coding capacity of its genome using overlapping reading frames. (pages 1306-1312)

In the 20th century, three pandemics of human influenza A virus occurred in 1918, 1957 and 1968; the one in 1918 was the most severe as 20 to 40 million people died worldwide. Pandemics are caused by influenza viruses with 'new' antigens. Such viruses can enter humans through the reassortment of genes between viruses that infect humans and those that infect animals or birds. (The 1957 and 1968 viruses acquired their genes from viruses that infected humans and an avian species ${ }^{1,2}$.) However, human pandemic influenza viruses may also arise in other ways. In 1997 in Hong Kong a lethal avian influenza virus was transmitted directly to humans from chickens and of the 18 known cases 6 were fatal. Despite the severity of the disease, relatively little is known about the viral proteins involved in pathogenicity.

\section{ROBERT A. LAMB \& MAKOTO TAKEDA}

The virulence of a virus is defined as its capacity to produce disease in a host ${ }^{3}$. For influenza viruses, due to the many factors involved it has proven difficult to elucidate the mechanism of virulence. The best understood determinant of virulence is whether the precursor of the hemagglutinin glycoprotein is cleaved intracellularly or extracellularly ${ }^{4}$ Other factors are also important; for example, virulence of the 1997 Hong Kong H5N1 influenza virus in mice is associated with the presence of lysine at residue 627 of the polymerase protein PB2 (ref. 5). The abilities of influenza virus to antagonize the host-defense interferon system ${ }^{6}$ and to either promote or inhibit apoptosis are also likely to contribute to the overall virulence of the virus. In this issue of Nature Medicine, Chen et al. ${ }^{7}$ describe a newly identified protein, derived from a second open reading frame (ORF) of $P B 1$ and show that the PB1-F2 protein associates with mitochondria and induces cell death.

The discovery of the new influenza virus protein is a classic example of how chance favors the prepared mind. The work began with an investigation of whether alternative reading frames of viral genes could be translated and the polypeptides used to generate antigenic peptides that mediate a cytotoxic T-lymphocyte (CTL) response-a $\mathrm{CD} 8^{+}$phenotype restricted by class I major histocompatibility (MHC) antigen. Infection of cells by influenza virus was chosen as a model system because of the authors' experience with the virus and 


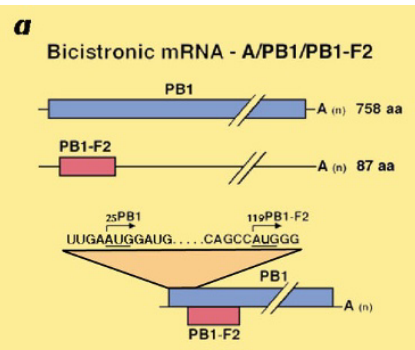

b

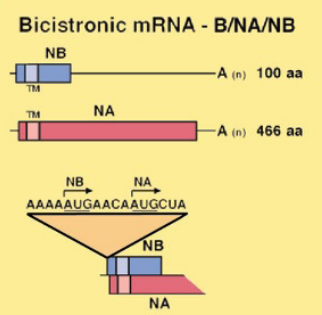

$\boldsymbol{d}$

Alternative splicing - A/M1/M2

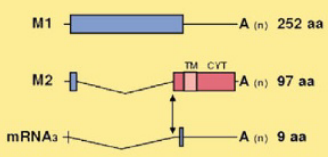

e

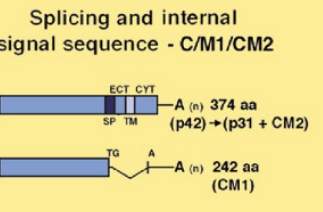

C

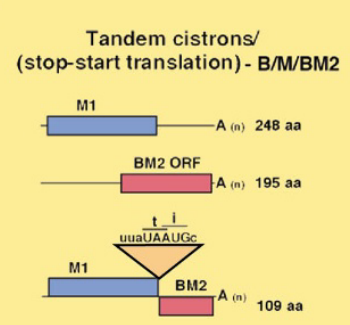

$\boldsymbol{f}$
Splicing - A/B/C/NS1/NEP(NS2)

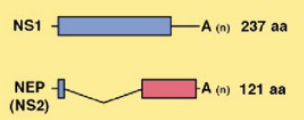

Fig. 1 Coding strategies of influenza A, B and C viruses to increase viral proteins. $\boldsymbol{a}$, The two ORFs in influenza A RNA segment 2 that encodes the polymerase PB1 and the newly described PB1-F2 proteins ${ }^{7}$. A single bicistronic mRNA is probably used for translation of both proteins. $\boldsymbol{b}$, The two ORFs in influenza B RNA segment 6 that encodes the integral membrane proteins NB and NA (NA is a neuraminidase; NB function is unknown.) It seems that a bicistronic mRNA is translated to yield NB and NA using two AUG codons separated by four nucleotides. The 2 discrete proteins are translated from overlapping ORFs. TM, transmembrane domain of integral membrane protein. $\boldsymbol{c}$, The 2 ORFs in influenza B RNA segment 7. A single mRNA contains 2 tandem cistrons, which encode the $\mathrm{M} 1$ and $\mathrm{BM} 2$ proteins. BM2 translation is thought to occur by a stop/start mechanism. The M1 (matrix) protein acts as a structural scaffold protein for the virion; BM2 function is unknown. t, termination codon for $\mathrm{M} 1$; i, initiation codon for BM2. d, Unspliced and alternatively spliced mRNAs derived from influenza A virus RNA segment 7. V-shaped lines indicate introns in the mRNAs. M1 functions as in c; M2 protein is an integral membrane protein with ion-channel activity, which is the target of the antiviral drug amantadine. CYT, cytoplasmic tail. $\boldsymbol{e}$, Unspliced and spliced mRNAs derived from influenza C RNA segment 6. M1 is translated from a spliced mRNA. The p42 precursor protein is translated from the unspliced linear transcript mRNA and is processed by a signal peptidase at an internal signal sequence to yield $\mathrm{p} 31$ (rapidly degraded) and the CM2 integral membrane protein (function unknown). SP, signal peptide; ECT, ectodomain; CYT, cytoplasmic tail. $f$, Unspliced and spliced mRNAs derived from RNA segment 8 of influenza $A$ and $B$ and RNA segment 7 of influenza C. (Influenza A is shown here.) NS1 is an antagonist of interferon; NEP (NS2) protein is implicated in viral-nucleocapsids $^{12}$. (Adapted from ref. 10, except for $a$ ).

because the viral genome size is manageable (that is, it consists of eight negativestranded RNA segments ranging from 2341 to 890 nucleotides in length for a total of about 13,588 nucleotides depending on the subtype ${ }^{8}$ ).

The authors performed a systematic search of the viral sequence in all reading frames for putative peptides with motifs containing primary binding sites for the mouse $\mathrm{MHC} \mathrm{H}-2 \mathrm{D}^{\mathrm{b}}$ peptide-binding groove, and they tested candidate synthetic peptides in CTL-stimulating assays. They identified a novel influenzavirus-specific peptide that bound to $\mathrm{H}-2 \mathrm{D}^{\mathrm{b}}$ with high affinity. This novel peptide, PB1-F2 ${ }_{62-70}$, was derived from a previously unrecognized second $(+1)$ ORF of PB1 (nucleotides 119-379; 87 amino acids for influenza virus strain $\mathrm{A} / \mathrm{PR} / 8 / 34)$. The peptide PB1-F2 $62-70$ was naturally expressed in influenza virusinfected cells and sensitized target cells against $\mathrm{CD}^{+} \mathrm{T}$ cells.
The PB1-F2 ORF is maintained in 64 out of 75 influenza A virus strains. Not surprisingly, given the lack of conservation, a genetic knockout of the PB1-F2 ORF yielded a viable virus for growth in tissue culture. (Genes that are non-essential for replication in tissue culture for many viruses, often play key roles in virus-host interactions, especially hostrange and pathogenicity.)

Expression and mutagenesis studies suggest, but do not prove, that there is a single mRNA that is bicistronic and is used to translate PB1 and PB1-F2. Examination of the subcellular distribution of PB1-F2 in virus-infected cells showed that the protein is detected predominantly in the mitochondria, but is also present in the nucleus and cytosol; however, when PB1-F2 was expressed from a vector it mostly localized to mitochondria. PB1-F2 expression caused alterations to mitochondrial morphology and immunogold-labeling electron mi- croscopy showed that PB1-F2 was almost exclusively associated with both the outer and inner mitochondrial membranes of transfected cells.

In a tour de force of synthetic peptide synthesis, the 87 residue $\mathrm{PB} 1-\mathrm{F} 2_{\text {syn }}$ protein was made, purified and microinjected into cells. PB1-F2 ${ }_{\text {syn }}$ localized to mitochondria and caused mitochondrial rounding, nuclear shrinkage and cell death. Remarkably, exogenous addition of PB1-F2 syn $_{\text {to cells also }}$ induced cell death. Thus, as suggested by the authors ${ }^{7}$, perhaps PB1-F2 forms membrane pores.

Influenza virus infection of monocytes causes apoptosis and one inducer of apoptosis is the viral non-structural protein, NS1 (ref. 9). Infection of a monocyte cell line and freshly isolated human monocytes with wild-type influenza virus or the PB1-F2 ORF knock-out virus showed a $50 \%$ increase in apoptosis by the wild-type virus. However, such changes in the degree of apoptosis were dependent on cell type and not observed in standard laboratory epithelial cells. Although more work needs to be done to understand the mechanism by which PB1-F2 mediates apoptosis, the celltype dependency may be important for pathogenicity. When PB1-F2 acts in cis it may disable virus-infected monocytes or other host innate immune cells whose normal role is to block viral infections. When PB1-F2 acts in trans (that is, when it is released from dying cells) it may inactivate the same types of host cells when they are recruited to the site of infection. Studies in appropriate model animal systems may help resolve some of these issues.

In addition to suggesting a mechanism by which the influenza A virus can inactivate the host immune response, the study by Chen et al. ${ }^{7}$ offers important insights into viral gene expression. The influenza A virus and the two closely related human viruses, influenza $\mathrm{B}$ and $\mathrm{C}$, use a remarkable collection of mechanisms to expand their genomecoding capacity beyond the one-geneper-genome RNA segment ${ }^{10}$ (Fig. 1). These strategies include translation of 
unspliced, spliced and alternatively spliced mRNAs, bicistronic mRNAs, as well as coupled stop/start translation of tandem cistrons. In many of these cases, protein translation occurs using overlapping reading frames. Influenza $\mathrm{C}$ virus also utilizes an unusual internal signal peptidase cleavage of a precursor protein to generate the integral membrane protein CM2 (Fig. 1e).

So far neither ribosomal frameshifting nor suppression of termination of translation has been identified for influenza viruses. Furthermore, the process of pseudo-templated addition of nucleotides to generate RNAs of different coding capacity, which was found for the V/P gene of paramyxoviruses ${ }^{11}$, has not been found for influenza viruses. Nonetheless, influenza viruses have not only increased the number of proteins encoded by genomes of limited size by using diverse coding strategies, but these strategies have also provided a means by which to regulate the expression of these proteins.

1. Wright, P.F. \& Webster, R.G. Orthomyxoviruses. in Fields Virology, 4th ed. (eds. Knipe, D.M. \& Howley, P.M.) 1533-1579 (Lippincott, Williams and Wilkins, Philadelphia, 2001)

2. Webster, R.G. Virology. A molecular whodunit. Science 293, 1773-1775 (2001).

3. Tyler, K.L. \& Nathanson, N. Pathogenesis of viral infections. in Fields Virology, 4th ed. (eds. Knipe, D.M. \& Howley, P.M.) 199-2431 (Lippincott, Williams and Wilkins, Philadelphia, 2001).

4. Steinhauer, D.A. Role of hemagglutinin cleavage for the pathogenicity of influenza virus. Virology 258, 1-20 (1999).

5. Hatta, M., Gao, P., Halfmann, P. \& Kawaoka, Y Molecular basis for high virulence of Hong Kong H5N1 influenza A viruses. Science 293, 1840-1842 (2001).

6. Garcia-Sastre, A. Inhibition of interferon-mediated antiviral responses by influenza $A$ viruses and other negative-strand RNA viruses. Virology 279, 375-384 (2001).

7. Chen, W. et al. A novel influenza A virus mitochondrial protein that induces cell death. Nature Med. 1306-1312 (2001).
8. Lamb, R.A. The influenza virus RNA segments and their encoded proteins. in Genetics of Influenza Viruses (eds. Palese, P. \& Kingsbury, D.W.) 26-69 (Springer, Vienna, 1983).

9. Schultz-Cherry, S., Dybdahl-Sissoko, N., Neumann, G., Kawaoka, Y. \& Hinshaw, V.S. Influenza virus NS1 protein induces apoptosis in cultured cells. J. Virol. 75, 7875-7881 (2001).

10. Lamb, R.A. \& Krug, R.M. Orthomyxoviridae: The viruses and their replication. in Fields Virology, 4th ed. (eds. Knipe, D.M. \& Howley, P.M.) 1487-1531 (Lippincott, Williams and Wilkins, Philadelphia, 2001).

11. Lamb, R.A. \& Kolakofsky, D. Paramyxoviridae: The viruses and their replication. in Fields Virology, 4th ed. (eds. Knipe, D.M. \& Howley, P.M.) 1305-1340 (Lippincott, Williams and Wilkins, Philadelphia, 2001).

12. Paragas, J. et al. Influenza $B$ and C virus NEP (NS2) proteins possess nuclear export activities. J. Virol. 75, 7375-7383 (2001)

Howard Hughes Medical Institute and

Northwestern University

Department of Biochemistry,

Molecular Biology and Cell Biology

Evanston, Illinois, USA

Email: ralamb@northwestern.edu

\title{
A fresh facet for ouabain action
}

\author{
Ouabain signaling through a plasma membrane can produce oscillations of intracellular calcium levels, resulting in \\ translocation of the NF- $\kappa B$ transcription factor into the nucleus and gene activation. This is a previously \\ unrecognized form of steroid action.
}

$\mathrm{O}$ ne of the oldest remedies for people with heart disease is treatment with plant-extract steroids of the digitalis family. Only in the past few years has it become apparent that mammals naturally produce a very similar compound, ouabain, a hormone thought to be synthesized in the adrenal glands. In humans (and dogs), ouabain concentrations increase rapidly in blood during physical exercise and decline quickly after rest. About half of people of European descent with a certain (low-renin) form of hypertension have abnormally high levels of ouabain, as do patients with congestive heart failure ${ }^{1,2}$.

So how might ouabain, and other steroids that have an effect on heart function, be acting at the cellular level? Writing in Proceedings of the National Academy Sciences, Aizman et al. ${ }^{3}$ describe experiments that reveal a new pathway: ouabain signaling through receptors on a cell's plasma membrane can induce slow oscillations of intracellular calcium levels and thereby activate a master regulator of cell activity - the transcription factor, NF$\kappa \mathrm{B}$. This finding is a notable addition to the emerging picture of how cardioactive steroids work.

The textbook explanations of ouabain action on vascular muscle center on

\section{Georgios SCHeINER-Bobis \& WILHELM SCHONER}

$\mathrm{Na}^{+} / \mathrm{K}^{+}$-ATPase, a protein that pumps sodium ions and is an integral part of a cell's plasma membrane. According to this explanation, inhibition of $\mathrm{Na}^{+} / \mathrm{K}^{+}$ATPase by ouabain leads to a rise in levels of cytosolic $\mathrm{Na}^{+}$and, through the involvement of $\mathrm{a} \mathrm{Na} / \mathrm{Ca}^{++}$-exchanger, to a rise in intracellular concentrations of $\mathrm{Ca}^{++}$. The upshot is increased contraction of vascular or heart muscle. But this view is clearly over-simplified, and has long been in need of refinement.

Aizman et ll. $^{3}$ show that, at low concentrations that only partially inhibit $\mathrm{Na}^{+} / \mathrm{K}^{+}$-ATPase, ouabain acts as a hormone and a signal transducer and activator for NF-kB. The course of events is shown as one of the pathways in Fig. 1. Slow $\mathrm{Ca}^{++}$oscillations are produced through the interplay of other proteins situated on the plasma membrane (storeoperated $\mathrm{Ca}^{++}$channels and L-type voltage-gated $\mathrm{Ca}^{++}$channels) and on the endoplasmic reticulum (the inositol 1,4,5-trisphosphate receptor). The result is translocation of NF- $\mathrm{KB}$ into the nucleus: NF- $\kappa \mathrm{B}$ regulates several genes involved in vascular physiology, and is also activated through angiotensin II-a hormone involved not only in controlling blood pressure but also in the pathogenesis of hypertension.

This finding that ouabain can activate NF- $\kappa B$ adds to a large body of data on intracellular signaling. For instance, partial inhibition of an ouabain-sensitive form of the sodium pump by nanomolar levels of ouabain may lead to $\mathrm{Ca}^{++}$transients in arteries evoked by a local rise of $\mathrm{Ca}^{++}$concentration within a specific, tiny cytosolic space between the plasma membrane and adjacent endoplasmic (or sarcoplasmic) reticulum. A local ouabain-dependent increase in levels of intracellular $\mathrm{Na}^{+}$ and $\mathrm{Ca}^{++}$within this space via the storeoperated channel and, eventually, the $\mathrm{Na}^{+} / \mathrm{Ca}^{++}$exchanger may augment the release of $\mathrm{Ca}^{++}$from intracellular stores. The secondary rise in the bulk concentration of $\mathrm{Ca}^{++}$may then lead to the increased muscle contraction ${ }^{2}$.

To date, however, no ouabain-dependent increase in $\left[\mathrm{Na}^{+}\right]$has been reported, possibly because it is difficult to detect small, local concentration changes that might occur in the space between plasma membrane and endoplasmic/sarcoplasmic reticulum. On the other hand, $\left[\mathrm{Ca}^{++}\right]$ elevation has been shown to occur in cardiac muscle cells ${ }^{4}$ : there, ouabain binding 